\title{
Uniportal subxiphoid video assisted thoracoscopy surgery approach for bilateral lesions
}

\author{
Balasubramanian Venkitaraman $^{1} \wedge$, Zhaolong Zeng $^{2}$, Lei Jiang $^{3} \wedge$, Giuseppe Aresu $^{4} \wedge$ \\ ${ }^{1}$ Division of Thoracic Oncology, Department of Surgical Oncology, Sri Ramachandra Institute of Higher Education and Research, Chennai, India; \\ ${ }^{2}$ Department of Thoracic Surgery, Hwa Mei Hospital, Ningbo Institute of Life and Health Industry, University of Chinese Academy of Sciences, \\ Ningbo, China; ${ }^{3}$ Department of Thoracic Surgery, Shanghai Pulmonary Hospital, Shanghai, China; ${ }^{4}$ Department of Thoracic Surgeon, Royal \\ Papworth Hospital, Cambridge, UK \\ Contributions: (I) Conception and design: B Venkitaraman, L Jiang, G Aresu; (II) Administrative support: Z Zeng, L Jiang; (III) Provision of study \\ materials or patients: Z Zeng, L Jiang, G Aresu; (IV) Collection and assembly of data: B Venkitaraman, Z Zeng, L Jiang; (V) Data analysis and \\ interpretation: B Venkitaraman, Z Zeng, L Jiang, G Aresu; (VI) Manuscript writing: All authors; (VII) Final approval of manuscript: All authors. \\ Correspondence to: Lei Jiang. Professor, Department of Thoracic Surgery, Shanghai Pulmonary Hospital, Shanghai, China. \\ Email: jiangleiem@aliyun.com.
}

Background: Minimal invasive surgery has become the standard of care for management of lung cancer. Increased use of high resolution CT scans and advent of screening programs has given rise to increased detection of incidental lesions. Conventional thoracoscopy does not provide for simultaneous, single approach surgery for bilateral lung lesions. Advent of subxiphoid technique helps in overcoming this problem. We describe our experience on uniportal subxiphoid thoracoscopy for bilateral lung lesions in a single stage surgery.

Methods: A retrospective analysis of prospectively maintained database done and data of patients undergoing single staged subxiphoid uniportal thoracoscopy surgery were analyzed. Preoperative parameters, intraoperative and postoperative data were analyzed. Data analysis was done using SPSS 17.0.

Results: A total of 37 patients were analyzed. Mean age of 56.76 years, with most patients in the 60-70 years age group. The preoperative mean BMI was 22.4 and mean FEV1\% was 92.187. Bilateral lobectomy in 6 patients, lobectomy with segmental resection was done in 14, bilateral segmental resection in 6 patients. Mean duration of surgery was 204.8 minutes with mean intraoperative blood loss of $115.9 \mathrm{~mL}$. There was one conversion to multiport VATS and two conversions to thoracotomy. The mean nodal yield was 10.33. Postoperative complications were seen in 7, three with Clavien-Dindo grade 3, two with grade 2 and two with grade 1 . The 30 -day mortality was zero. The postoperative duration of hospital stay and complications were significantly more common after bilateral segmental resections $(\mathrm{P}<0.01)$. Postoperative histology revealed invasive adenocarcinoma (at least in one specimen) in $51.3 \%$ and in both in $13.5 \%$. Bilateral adenocarcinoma in situ (Tis) was found in $13.5 \%$ and adenocarcinoma in situ associated with minimal invasive adenocarcinoma was found in $5.4 \%$.

Conclusions: Uniportal Subxiphoid thoracoscopy offers the advantage of bilateral access through a single incision and is found to be technically sound and safe with comparable postoperative outcomes. Simultaneous bilateral lobectomy tends to have higher rate of complications and thus should be done after proper patient selection.

Keywords: Uniportal video assisted thoracoscopy surgery (VATS); subxiphoid uniportal VATS; bilateral lung surgery

^ ORCID: Balasubramanian Venkitaraman, 0000-0002-3983-944X; Lei Jiang, 0000-0002-5687-8037; Giuseppe Aresu, 0000-0001-5708-8195. 
Received: 18 May 2020; Accepted: 07 October 2020; Published: 20 January 2021.

doi: 10.21037/jovs-2020-14

View this article at: http://dx.doi.org/10.21037/jovs-2020-14

\section{Introduction}

Advent of minimal invasive technique has revolutionized thoracic surgery, over the last 2 decades. Video assisted thoracoscopy surgery (VATS) made thoracic surgery less morbid and reduced the overall treatment duration and complications with similar oncological outcomes $(1,2)$.

On the other side, the increased use of screening CT scan in high risk population made it feasible to detect lesions at an early stage and reduce related mortality (3). However, detection of additional multiple lung pathologies have also increased with the use of high definition CT scan. Some of these lesions are difficult to be characterized by imaging alone and are also not easily amenable for image guided biopsy. Management of such multiple lung lesions, especially those detected in lung cancer pose a great challenge in staging and management. Current guidelines for management of lung cancer, including NCCN and IASLC guidelines have not addressed this adequately and it is yet being debated, whether to consider them as metastasis from the primary lung lesion or as synchronous multiple primary lung lesions. A few criteria have been described in literature for classification of primary multiple lung cancers $(4,5)$, however these were published at a time when the CT scan was less developed. Recent evidence suggests that aggressive surgical resection yields the best possible outcomes in select such patients (6,7). Minimally invasive surgery with its proven advantage over conventional thoracotomy can be increasingly used when bilateral simultaneous surgical resection is required.

Uniportal VATS (U-VATS) for management of lung cancer have been found to be safe and oncologically sound (8) however the Uniportal Subxiphoid approach offers the extra benefit of providing a bilateral access using a single incision and avoiding intercostal nerve injury (9). Bilateral lung surgery using Sub Xiphoid U-VATS has-been described in literature (10-12).

In this article we describe our experience in uniportal subxiphoid synchronous pulmonary resection for bilateral lung lesions, performed at our tertiary care centre. We present the following article in accordance with the STROBE reporting checklist (available at https://jovs. amegroups.com/article/view/10.21037/jovs-2020-14/rc).

\section{Methods}

We performed a retrospective analysis, of prospectively maintained database of patient undergoing surgery at our tertiary care hospital, between October 2014 and December 2017. Medical records of patients who underwent bilateral simultaneous subxiphoid pulmonary resection were analyzed after obtaining permission from the institute ethics committee. All patients, as per routine protocol had been evaluated with high resolution contrast CT of the chest and PET CT (whenever feasible). The lesions identified were classified into pure ground glass opacities, mixed ground glass lesion, solid nodules or mass lesion. Wherever possible image-guided or bronchoscopy guided biopsy of the lesion was performed and in patients where the same was not possible intraoperative fast frozen histology examination was done to confirm the presence of malignancy. Patients having multiple bilateral lung lesions, with no significant lymph nodal involvement and no evidence of metastatic disease elsewhere were considered for surgery after pulmonary function and cardiac function work up and fitness. Selection criteria for simultaneous procedure included forced expiratory volume in $1 \mathrm{~s}$ (percentage of predicted) (FEV1\%) was more than $60 \%$ and predicted postoperative $\mathrm{FEV} 1$ was more than 1.0 liter, with no significant cardiac co morbid conditions.

Selection of surgical procedure-depended on the preoperative imaging and varied from wedge resection through anatomical segmental resection to lobectomy. We did not undertake any pneumonectomy combined with contralateral lung resection in a single sitting.

Surgeries, performed by a single attending consultant, were taken up for this analysis. Patients' demographic data, preoperative patients' characteristics, intraoperative parameters and immediate postoperative outcomes were obtained from the data base and analyzed using SPSS 17.0.

The study was conducted in accordance with the Declaration of Helsinki (as revised in 2013). The study was approved by institutional review board of Shanghai Pulmonary Hospital (Approval number: K17-160). Individual Consent was waived off as this was a retrospective analysis and data was accessed after masking the patient's identity. 

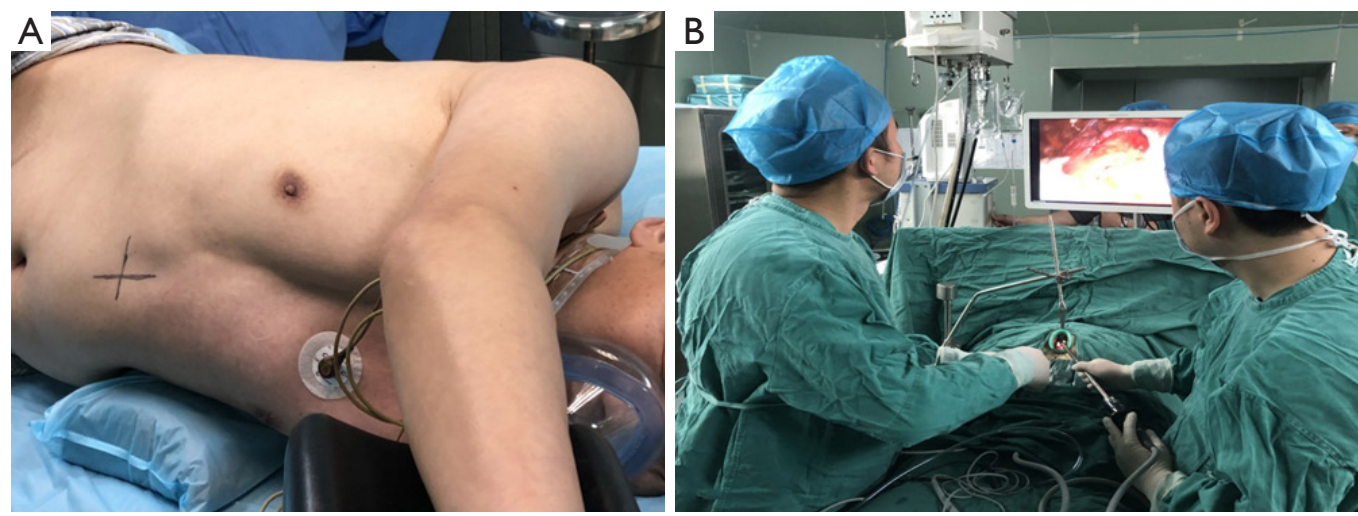

Figure 1 Operative positioning. (A) Positioning of patient with incision marking; (B) intraoperative position of surgeon and assistant and the visual module.

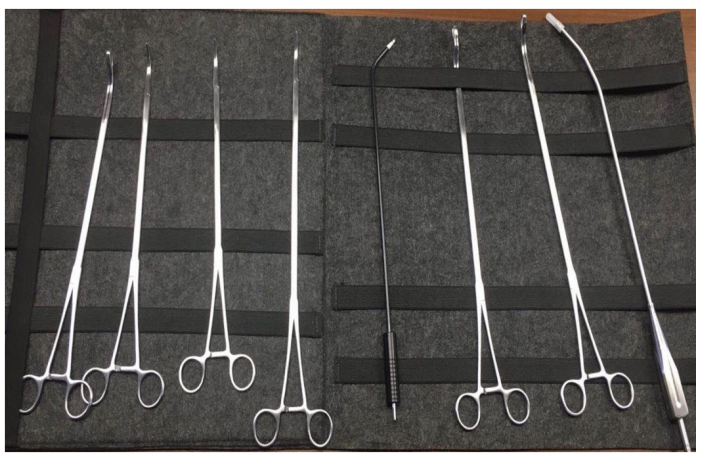

Figure 2 Instruments for subxiphoid uniportal video assisted thoracoscopy surgery (VATS).

\section{Surgical technique}

All patients were given general anesthesia using a double lumen endotracheal tube for obtaining single lung ventilation. The patients were placed in supine position, aseptic methods used for painting and draping was done. A $4 \mathrm{~cm}$ horizontal skin incision (vertical if the costal margins were close) is given in the subxiphoid region (Figure 1). Subcutaneous tissue and rectus abdominis muscle were separated by blunt dissection, staying extra peritoneal through-out the course. Sub-costal tunnel was created and mediastinal pleura were opened up in the region of cardiophrenic angle. Mediastinal fat was dissected out and one side pleural cavity was entered. A wound protector was placed at the site. Using a $10 \mathrm{~mm}$ 30-degree thoracoscope, pleural cavity was evaluated. Surgery was performed using specialized uniportal VATS instruments (Figure 2). The hilar structures were defined and as per the surgery, required hilar vasculature was taken down with articulating staplers (Covidien, Mansfield, USA or Ethicon Endo- Surgery INC, USA). Specimen was delivered using a specimen bag (or locally engineered glove bag) (Figure 3) (Videos 1,2). Post intended resection; the lung was re-inflated, to check for air leak. The contralateral lung was deflated and surgical procedure on the contralateral side was performed. In patient with a diagnosed primary malignancy of lung or in those patients were the intraoperative frozen was suspicious of invasive malignancy, formal lymph nodal dissection was performed. After completion of procedure, chest drain was placed through the same single incision (Figure 4).

\section{Results}

\section{Demography}

We identified a total of 37 cases, where bilateral pulmonary lesions were detected on preoperative evaluation and subxiphoid bilateral simultaneous pulmonary resection was done. The mean age of our patients was $56.76 \pm 10.93$ years (ranging from 27 to 74 years). Most common age group was 60 to 70 years $(41 \%)$, followed by 50 to 60 years $(25.6 \%)$. A smoking history was found only in a small percentage accounting for $18.9 \%$. Majority of the patients were females (62.2\%). The mean preoperative body mass index (BMI) measurement was $22.4 \pm 3.05 \mathrm{~kg} / \mathrm{m}^{2}$, ranging from 16.6 to $27.3 \mathrm{~kg} / \mathrm{m}^{2}$. Majority of the patients were in the BMI range of $20-25 \mathrm{~kg} / \mathrm{m}^{2}(51.4 \%)$. In the preoperative pulmonary function test, the mean FEV1\% was $92.187 \pm 17.47$. The lowest value was $69.5 \%$ (Table 1 ). 

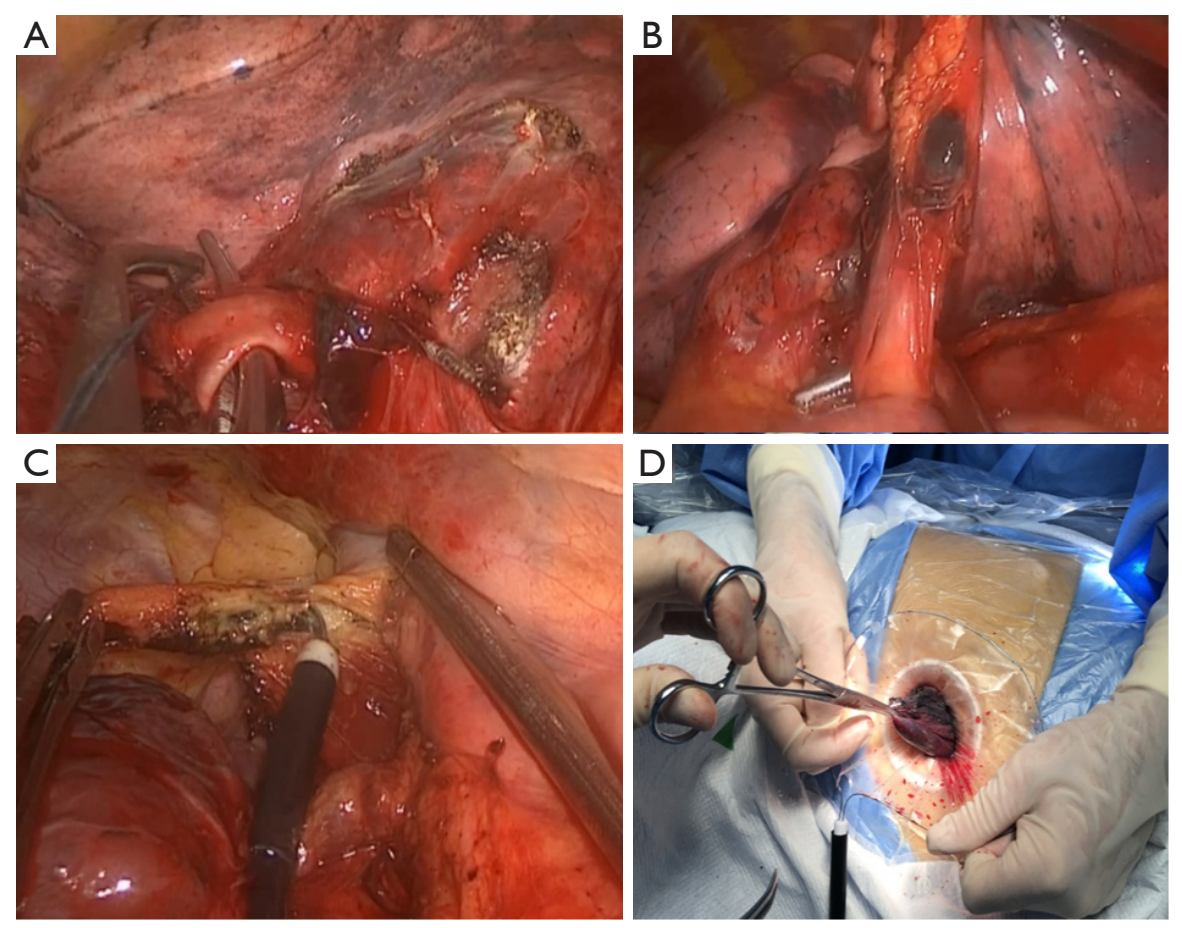

Figure 3 Intraoperative images. (A,B) Dissection of pulmonary vessels; (C) nodal dissection; (D) specimen retrieval in specimen bag.

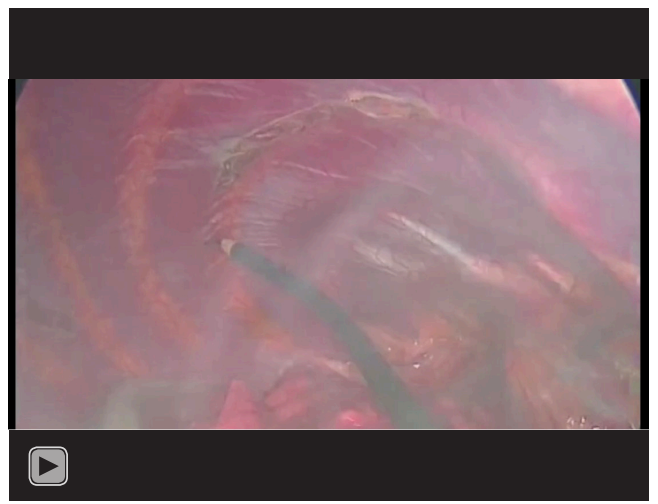

Video 1 Bilateral subxiphoid.

\section{Intra operative data}

Surgical procedures ranged from wedge resection to anatomical pulmonary resections (combinations of techniques were used) based on the preoperative evaluation and after the multidisciplinary board discussion and concurrence. All the 37 surgeries included in this analysis were done as single staged procedure. Bilateral lobectomy was performed in 6 patients, while lobectomy was combined with wedge resection in 2 patients and with anatomical

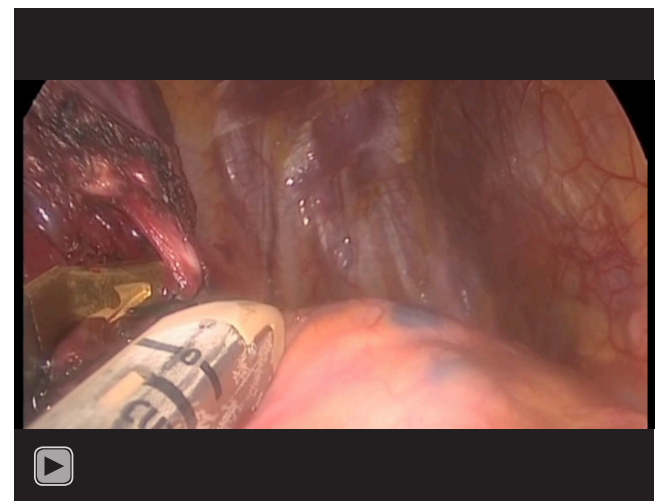

Video 2 Subxiphoid bilat segm short.

segmentectomy in 14 patients. A bilateral segmental resection was performed in 6 patients, in 3 patients segmental resection was combined with wedge resection (Table 2).

The mean duration of surgery was $204.8 \pm 71.7$ minutes. The mean operative blood loss was $115.95 \pm 53.98 \mathrm{~mL}$. There were 3 conversions in the surgery group (additional one intercostals port -1 , thoracotomy -2 ). All patients were extubated on table and kept in postoperative recovery room. 


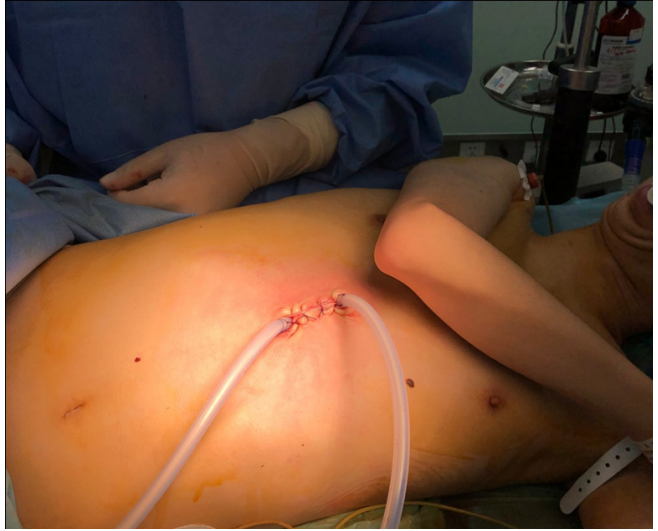

Figure 4 Postoperative wound of subcostal uniportal video assisted thoracoscopy surgery (VATS).

\section{Postoperative outcomes}

The mean duration of postoperative stay in the hospital was $6.78 \pm 6.8$ days (ranging from 2 to 41 days). Postoperative complications were seen in 7 patients. Three were ClavienDindo grade 3, one patient developed cerebrovascular infarction with residual paralysis, two patient developed pulmonary infection needing ICU readmission and mechanical ventilation. Two patients with Clavien-Dindo grade 2 complications-prolonged ICD drainage with collapse consolidation of lung, requiring long hospital stay and supplemental oxygen. Two patients had prolonged air leak requiring longer duration of chest drain placement. There was no postoperative 30-day mortality.

The postoperative stay in patients undergoing single stage bilateral lobectomy was $15.5( \pm 14.6)$ days compared $5.10( \pm 1.77)$ in other patients. On independent sample $T$ test, this was found to be statistically significant $(\mathrm{P}<0.01)$ with a $95 \%$ CI (5.2-15.6). The postoperative complications were also found to be more in patients undergoing bilateral lobectomy compared to others, showing statistical significance (Fisher's exact test $\mathrm{P}=0.007$ ).

\section{Histopathology}

Invasive adenocarcinoma was seen in $51.3 \%$ of patients, in at least in one specimen. Bilateral invasive adenocarcinoma was seen in $13.5 \%$. Invasive adenocarcinoma was associated with minimally invasive adenocarcinoma (MIA) of the other side in $5.4 \%$, adenocarcinoma in situ in $18.9 \%$ and benign pathology in $13.5 \%$. Bilateral adenocarcinoma in situ was found in $13.5 \%$ and associated with MIA in
Table 1 Demographic data

\begin{tabular}{lc}
\hline Characteristic & Data \\
\hline Age (years), mean \pm SD & $56.76 \pm 10.9$ \\
Sex, $\mathrm{n}(\%)$ & \\
Male & $14(37.8)$ \\
Female & $23(62.2)$ \\
BMl $\left(\mathrm{Kg} / \mathrm{m}^{2}\right)$, mean $\pm \mathrm{SD}$ & $22.24 \pm 3.05$ \\
Range & $16.59-27.25$ \\
Preoperative pulmonary function test, mean $\pm \mathrm{SD}$ & \\
FVC\% & $95.77 \pm 16.06$ \\
FEV1\% & $92.19 \pm 17.5$ \\
Positive Smoking history, \% & 8.9 \\
\hline
\end{tabular}

Table 2 Operative details

\begin{tabular}{lc}
\hline Surgery performed & Data \\
\hline Bilateral Lobectomy, N (\%) & $6(16.2)$ \\
Lobectomy + segmentectomy, N (\%) & $14(37.8)$ \\
Lobectomy +wedge resection, N (\%) & $2(5.4)$ \\
Bilateral segmentectomy, N (\%) & $6(16.2)$ \\
Segmentectomy + Wedge resection, N (\%) & $3(8.1)$ \\
Bilateral Wedge Resection, N (\%) & $6(16.2)$ \\
Nodal Dissection, mean \pm SD & \\
Lymph node station & $4.07 \pm 0.96$ \\
Lymph nodes removed & $10.33 \pm 2.7$ \\
Surgery Duration (minutes), mean \pm SD & $204.83 \pm 71.7$ \\
Operative blood loss (mL), mean \pm SD & $115.95 \pm 53.98$ \\
\hline
\end{tabular}

$5.4 \%$, with benign pathology in $2.7 \%$. One patient was operated for metastasectomy and bilateral tumors were metastatic deposits. Three patients underwent surgery for only bullectomy and in 2 other patients bilateral lesions were benign. (Table 3). The mean number of lymph nodes dissected was $10.33( \pm 2.7)$ and the median number of nodal stations sampled was 4 .

\section{Discussion}

Multiple primary lung cancer accounts for $0.8-8.4 \%$ of lung cancers among the Asian population $(13,14)$. 
Table 3 Postoperative histology

\begin{tabular}{lc}
\hline Histology & $\mathrm{N}$ \\
\hline Invasive tumor* & 19 \\
Bilateral invasive tumor & 5 \\
Invasive tumor with MIA & 2 \\
Invasive tumor with AIS & 7 \\
Invasive tumor with & (13.5) (Tuberculous, 2; cryptococcus, 1; \\
benign pathology & fibrosis, 2) \\
Minimally invasive tumor & 2 \\
+ AlS & 2 (AAH, 2) \\
MIA + benign & 5 Tis \\
Bilateral AIS & 3 Tis (AAH, 1; Fibrosis, 2) \\
AIS + benign & 3 \\
Bullae & 2 \\
Benign & 1 \\
Metastatic disease & \\
(secondary) &
\end{tabular}

${ }^{*}$, invasive tumor in at least one of the two specimens. MIA, minimally invasive adenocarcinoma; AIS, adenocarcinoma in situ; AAH, atypical adenomatous hyperplasia.

Increased use of high definition CT images and PET scan has resulted in increased detection of additional lung lesions in patients with lung cancer, however little has been described about the management of imaging detected lesion in such patients. The updates from the Fleischner Society recommendation in patients with multiple lung nodules with a dominant nodule, suggest that the dominant nodule determines the management. For the associated GGO, surgical management is recommended (15). In another new change in the management of lung cancer, the new WHO classification reclassifies the previously described bronchoalveolar carcinoma into further subtypes: adenocarcinoma in situ, MIA and invasive adenocarcinoma with the former two having near $100 \%$ survival after surgical resection $(16,17)$.

Uniportal VATS resection is being used more frequently in the management of lung cancers, with proven procedural safety and feasibility in patients with lung cancer (8). The advantage of uniportal over conventional multiportal thoracoscopy is the avoidance of injury to intercostal nerve at multiple places and resultant increased pain and paresthesia. Subxiphoid Uniportal VATS scores over the intercostals uniportal VATS in totally avoiding intercostal nerve injury, approach of bilateral lung lesions using a single incision. There have been a few reports of uniportal subxiphoid approach for bilateral lung lesions (10-12).

Wang et al. described the data on subxiphoid uniportal VATS in bilateral lung lesion in 11 patients. In their series, the mean age of the population involved was 42.6 years and 20-30 years and 40-50 years were the most common age group (27.3\% respectively) (11). In the series by Cai et al. comparing uniportal intercostal Uniportal VATS bilateral lung resection with sub Xiphoid uniportal resection (10), the mean age was $53.5 \pm 13.5$ years. In our study, the mean age was $56.76 \pm 10.9$ years and most common age group was $60-70$ years (41.0 years). Majority of population in the Wang et al. series were men (54.5\%) while in Cai et al. and in our results majority were women $(57.1 \%$ and $62.2 \%$ respectively). Most of the patients in our study were non obese patients (mean BMI 22.24) similar to Cai et al. (10) and the preoperative $\mathrm{FEV} 1 \%$ was also comparable to Cai et al. study (92.19 vs. 90.5 respectively).

Most of the procedures in Wang et al. series were bilateral non anatomical lung resections (wedge resection) with only 3 patients receiving anatomical lung resection that too on one side only. Cai et al. bilateral wedge resection in $32.1 \%$, lobectomy with wedge resection in $28.6 \%$ while bilateral anatomical lung resection (lobectomy +segmentectomy/bilateral segmentectomy) were described in only $28.5 \%$. In our series majority of surgeries (70.2\%) were bilateral anatomical lung resection (bilateral lobectomy: $16.2 \%$; bilateral segmentectomy: $16.2 \%$; lobectomy with segmentectomy: $37.8 \%)$. Our series is the first to describe the outcomes of bilateral lobectomy (in 6 cases). The mean operative time in our series was 204.8 minutes and the average amount of intraoperative blood loss was $115.9 \mathrm{~mL}$, which was comparatively lower than Cai series (212.3 minutes and $190.9 \mathrm{~mL}$ respectively).

There were three conversions from uniportal subxiphoid in our series, similar to Cai et al. However, we did not experience any re exploration. Postoperative stay was comparable at $6.78 v$ vs. 6.4 days respectively (10). Postoperative complications were $18.9 \%$, higher than in the other series $(10,11)$. Further analysis of our study population, showed the complication rates to be more common among bilateral lobectomy compared to other bilateral resection group (statistically significant). This was similar to the observation in the Cai et al. series.

On Histopathological examination of the specimen, invasive carcinoma was detected in $51.3 \%$ population almost similar to the Cai et al. series (10), while it was 
lower in Yang et al. series (11). Invasive adenocarcinoma was the most common histology in our population, similar to other studies $(10,11,18)$. Bilateral invasive carcinoma was found in 5 patients, while in $24.3 \%$ it was associated with MIA or Adenocarcinoma in situ. None of the patients with invasive tumours had nodal involvement. The median number of lymph node stationed sampled and nodes removed were compared to unilateral intercostal uniportal VATS (8). Highest stage of patient in our series as per the current AJCC 10th edition was T1 (40.5\%) comparable to the literature $(10,14)$. In this series, most of the patients with bilateral lung lesions had a coexisting preinvasive or microinvasive disease similar to Nakata et al. where well differentiated adenocarcinoma with bronchoalveolar pattern was the commonest observed subtype (39.7\%) (14). This supports the fact that aggressive surgical intervention is the key for management of synchronous bilateral lung lesions in select patients $(6,7,10,18)$.

Our study has a few short-comings, it was a retrospective analysis and long term follow up was not available to be included. The latter will help in survival analysis and help in better selection of surgery as modality for bilateral lung cancers. Also, a larger study with more number of patients will help in better understanding, however it may be difficult to perform one, given the lower incidence of bilateral lung tumours.

\section{Conclusions}

Aggressive surgical approach may be considered in patients with multiple synchronous pulmonary tumours, after proper patient selection. Bilateral synchronous anatomical lung resection is feasible and safe with minimal postoperative complications. With segmentectomy becoming more widely accepted for management of small tumours, bilateral segmental resection or segmental resection in combination with lobectomy, will not compromise the postoperative lung reserve, also address the tumour oncologically.

\section{Acknowledgments}

Funding: None.

\section{Footnote}

Provenance and Peer Review: This article was commissioned by the Guest Editor (Federico Rea) for the series "Alternative Surgical Approaches for Challenging Cases in Thoracic Surgery" published in Fournal of Visualized Surgery. The article has undergone external peer review.

Reporting Checklist: The authors have completed the STROBE reporting checklist. Available at https://jovs. amegroups.com/article/view/10.21037/jovs-2020-14/rc

Data Sharing Statement: available at https://jovs.amegroups. com/article/view/10.21037/jovs-2020-14/dss

Conflicts of Interest: All authors have completed the ICMJE uniform disclosure form (available at https:// jovs.amegroups.com/article/view/10.21037/jovs-202014/coif). The series "Alternative Surgical Approaches for Challenging Cases in Thoracic Surgery" was commissioned by the editorial office without any funding or sponsorship. The authors have no other conflicts of interest to declare.

Ethical Statement: The authors are accountable for all aspects of the work in ensuring that questions related to the accuracy or integrity of any part of the work are appropriately investigated and resolved. The study was conducted in accordance with the Declaration of Helsinki (as revised in 2013). The study was approved by institutional review board of Shanghai Pulmonary Hospital (Approval number: K17-160). Individual Consent was waived off as this was a retrospective analysis and data was accessed after masking the patient's identity.

Open Access Statement: This is an Open Access article distributed in accordance with the Creative Commons Attribution-NonCommercial-NoDerivs 4.0 International License (CC BY-NC-ND 4.0), which permits the noncommercial replication and distribution of the article with the strict proviso that no changes or edits are made and the original work is properly cited (including links to both the formal publication through the relevant DOI and the license). See: https://creativecommons.org/licenses/by-nc-nd/4.0/.

\section{References}

1. Kirby TJ, Mack MJ, Landreneau RJ, et al. Lobectomy-video-assisted thoracic surgery versus muscle-sparing thoracotomy. A randomized trial. J Thorac Cardiovasc Surg. 1995;109:997-1001; discussion 1001-2.

2. Zhang W, Wei $Y$, Jiang H, et al. Video-Assisted Thoracoscopic Surgery Versus Thoracotomy Lymph Node Dissection in Clinical Stage I Lung Cancer: A 
Meta-Analysis and System Review. Ann Thorac Surg 2016;101:2417-24.

3. National Lung Screening Trial Research Team, Aberle DR, Adams AM, et al. Reduced lung-cancer mortality with low-dose computed tomographic screening. N Engl J Med. 2011;365:395-409.

4. Martini N, Melamed MR. Multiple primary lung cancers. J Thorac Cardiovasc Surg 1975;70:606-12.

5. Antakli T, Schaefer RF, Rutherford JE, Read RC. Second primary lung cancer. Ann Thorac Surg 1995;59:863-6; discussion 867.

6. Chen C, Huang X, Peng M, et al. Multiple primary lung cancer: a rising challenge. J Thorac Dis 2019;11:S523-36.

7. Yu YC, Hsu PK, Yeh YC, et al. Surgical results of synchronous multiple primary lung cancers: similar to the stage-matched solitary primary lung cancers? Ann Thorac Surg. 2013;96:1966-74.

8. Venkitaraman B, Lei J, Liang W, et al. Uniportal videoassisted thoracoscopy surgery in lung cancer: largest experience. Asian Cardiovasc Thorac Ann 2019;27:559-64.

9. Song N, Zhao DP, Jiang L, et al. Subxiphoid uniportal video-assisted thoracoscopic surgery (VATS) for lobectomy: a report of 105 cases. J Thorac Dis 2016;8:S251-7.

10. Cai H, Xie D, Al Sawalhi S, et al. Subxiphoid versus intercostal uniportal video-assisted thoracoscopic surgery for bilateral lung resections: a single-institution experience. Eur J Cardiothorac Surg 2020;57:343-9.

11. Yang X, Wang L. Subxiphoid uniportal video-assisted

doi: 10.21037/jovs-2020-14

Cite this article as: Venkitaraman B, Zeng Z, Jiang L, Aresu G. Uniportal subxiphoid video assisted thoracoscopy surgery approach for bilateral lesions. J Vis Surg 2021;7:4. thoracoscopic surgery for synchronous bilateral lung resection. Postgrad Med 2018;130:142-5.

12. Aresu G, Weaver H, Wu L, et al. Uniportal subxiphoid video-assisted thoracoscopic bilateral segmentectomy for synchronous bilateral lung adenocarcinomas. J Vis Surg 2016;2:170.

13. Wu SC, Lin ZQ, Xu CW, et al. Multiple primary lung cancers. Chest 1987;92:892-6.

14. Nakata M, Sawada S, Yamashita M, et al. Surgical treatments for multiple primary adenocarcinoma of the lung. Ann Thorac Surg 2004;78:1194-9.

15. Naidich DP, Bankier AA, MacMahon H, et al. Recommendations for the management of subsolid pulmonary nodules detected at CT: a statement from the Fleischner Society. Radiology 2013;266:304-17.

16. Travis WD, Brambilla E, Noguchi M, et al. International association for the study of lung cancer/American thoracic society/European respiratory society international multidisciplinary classification of lung adenocarcinoma. J Thorac Oncol 2011;6:244-85.

17. Kadota K, Villena-Vargas J, Yoshizawa A, et al. Prognostic significance of adenocarcinoma in situ, minimally invasive adenocarcinoma, and nonmucinous lepidic predominant invasive adenocarcinoma of the lung in patients with stage I disease. Am J Surg Pathol 2014;38:448-60.

18. Jung EJ, Lee JH, Jeon K, et al. Treatment outcomes for patients with synchronous multiple primary non-small cell lung cancer. Lung Cancer 2011;73:237-42. 